\title{
Compact Source of EPR Entanglement and Squeezing at Very Low Noise Frequencies
}

\author{
J. Laurat, ${ }^{1}$ T. Coudreau, ${ }^{1,2, *}$ G. Keller,${ }^{1}$ N. Treps,${ }^{1}$ and C. Fabre ${ }^{1}$ \\ ${ }^{1}$ Laboratoire Kastler Brossel, UPMC, Case 74, 4 Place Jussieu, 75252 Paris cedex 05, France \\ ${ }^{2}$ Laboratoire Matériaux et Phénomènes Quantiques, \\ Université Denis Diderot, 2 Place Jussieu, 75251 Paris cedex 05, France
}

\begin{abstract}
We report on the experimental demonstration of strong quadrature EPR entanglement and squeezing at very low noise sideband frequencies produced by a single type-II, self-phase-locked, frequency degenerate optical parametric oscillator below threshold. The generated two-mode squeezed vacuum state is preserved for noise frequencies as low as $50 \mathrm{kHz}$. Designing simple setups able to generate non-classical states of light in the $\mathrm{kHz}$ regime is a key challenge for high sensitivity detection of ultra-weak physical effects such as gravitational wave or small beam displacement.
\end{abstract}

PACS numbers: 03.67.Mn, 04.80.Nn, 42.50 Dv, 42.50.Lc, 42.65.Yj

Since the pioneering work of Caves [1] which showed that it is possible to improve the sensitivity of interferometric measurements by the use of squeezed light, and its experimental demonstrations 2], various protocols involving squeezed light have been discussed in order to beat the standard quantum limit in gravitational wave detectors [3]. As next generations of gravitational wave detectors will be designed to be shot noise limited in the acoustic band from $10 \mathrm{~Hz}$ to $10 \mathrm{kHz}$, such techniques appear as quite promising ways to improve their sensitivity. Recently, a squeezing-enhanced power-recycled Michelson interferometer has been experimentally demonstrated and signal-to-noise ratio improvement obtained [4]. However, the injected squeezing bandwidth lies around $5 \mathrm{MHz}$ and not in the frequency band of gravitational waves. A source of low frequency squeezing is thus a key point for the implementation of future squeezed-input interferometers.

More generally, many high sensitivity measurements performed at low modulation frequency can benefit from such a device. In 5], a "quantum laser pointer" has been experimentally demonstrated and an improvement of modulated small displacement measurements in two orthogonal directions in the transverse plane has been reported. Improved beam positioning sensitivity below the shot noise limit is obtained at the frequency where squeezing is available, a few $\mathrm{MHz}$ in the case of this experiment. Two sources of low frequency squeezing are needed to apply this promising displacement measurement technique to actual instruments where frequency modulation is generally low. This could be applied for instance to AFM microscopy in tapping mode where cantilever oscillates at its resonant frequency which is typically a few hundreds of $\mathrm{kHz}$. Such a squeezing source should also improve the thermo-optical spectroscopy technique called "mirage effect" [6], which enables the measurement of very weak absorption: a thermo-optical modulation on a sample induces a periodic refractive index gradient and results on a low frequency modulated probe beam deflection.

Broadband and low frequency squeezing is also very useful even if the information is not carried by a single frequency modulation. When a beam is detected during a finite time, the signal-to-noise ratio depends on the noise in an extended range of sideband frequencies. Broadband squeezing with a cut-off frequency as low as possible is thus required. A great number of measurements can be improved in that way, for instance the detection of weak pulsed signals or the reduction of the bit error rate in the readout of digitized optical information.

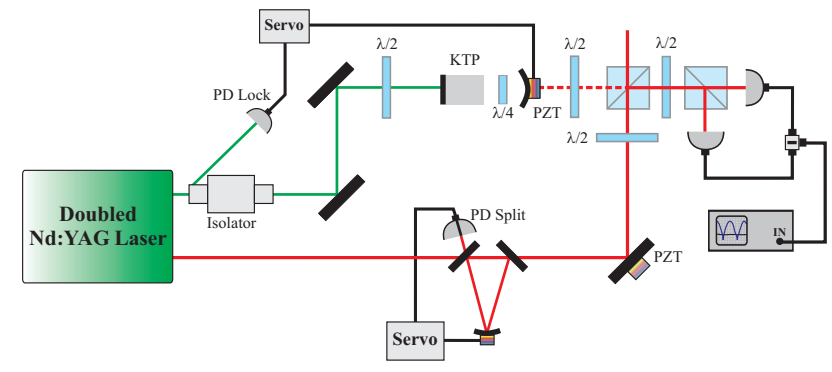

FIG. 1: Experimental layout. A continuous frequencydoubled Nd:YAG laser pumps below threshold a frequency degenerate type-II phase-matched OPO with a quarter-wave plate inserted inside the cavity. The generated two-mode state is characterized by homodyne detection and the difference of the photocurrents is sent onto a spectrum analyser. PD Lock: FND-100 photodiode for locking of the OPO. PD Split: split two-element InGaAs photodiode for tilt-locking of the filtering cavity.

In 7], quantum noise reduction on the intensity difference of twin beams has been observed down to $90 \mathrm{kHz}$. However, only two experiments to date have demonstrated continuous-wave squeezing at low frequencies. Both experiments are based on an optical noise cancellation scheme where sources of squeezing are inserted within a Mach-Zehnder interferometer: squeezing has been obtained around $220 \mathrm{kHz}$ with a pair of independent optical parametric amplifiers (OPA) [8] and very recently around $100 \mathrm{kHz}$ with a single OPA [9]. This frequency range is rather unusual in the experimental quantum optics field where non-classical properties are 
generally observed in the $\mathrm{MHz}$ range due to the presence of large classical noise at lower frequencies. In this letter, we report on what is to our knowledge the first experimental demonstration of very low frequency continuouswave squeezing without need of optical noise cancellation scheme. Broadband vacuum squeezing is observed for frequencies down to $50 \mathrm{kHz}$. Furthermore, our setup generates not only single-mode squeezing but also EPR entanglement which is a basic requisite in quantum information protocols such as teleportation, dense-coding or optical-atomic interfacing [10].

Our setup relies on a frequency degenerate type-II phase-matched optical parametric oscillator (OPO) below threshold, in which a quarter-wave plate inserted in the cavity adds a linear coupling between the signal and idler fields. In this letter, we focus on the case where the plate is rotated by a very small angle (smaller than $0.02^{\circ}$ ) relative to the principal axis of the non-linear crystal. In such a configuration, the signal and idler modes are entangled: they show quantum correlations and anticorrelations on orthogonal quadratures for noise frequencies inside the bandwidth of the cavity. This non-classical behavior exists also without the plate. However, the linear coupling - even for a plate rotated by a very small angle - facilitates the finding of experimental parameters for which entanglement is observed. When our setup is operated above threshold, frequency degenerate operation is obtained in a small locking zone and not only for a precise value of experimental parameters 11], which corresponds also to the required parameters below threshold. When this zone is found, the OPO can be operated below threshold and the entanglement is maximized by fine tuning of the crystal temperature. Furthermore, the degenerate operation with bright beams opens the possibility to match the homodyne detection without infrared injection of the OPO. In prior experiments with KTP crystals and green pump, pairing of crystals in order to compensate walk-off [2] or using $\alpha$-cut KTP for noncritical phase matching and frequency-doubled Nd:YAP laser [12] were necessary to generate entangled states of light.

Instead of directly measuring the quantum correlations and anti-correlations of the signal and idler fields, $A_{1}$ and $A_{2}$, we characterize the noise of the superposition modes oriented $\pm 45^{\circ}$ from the axes of the crystal

$$
A_{+}=\frac{A_{1}+A_{2}}{\sqrt{2}} \quad \text { and } \quad A_{-}=\frac{A_{1}-A_{2}}{\sqrt{2}}
$$

These two modes have squeezed fluctuations due to the correlations and anti-correlations between signal and idler fields. The amount of entanglement between signal and idler can be inferred from the amount of squeezing available on these superposition modes. We have developed in 13] a theoretical study of this original device named "self-phase-locked OPO" in the above threshold regime. Below threshold, theoretical and experimental behavior for various birefringent plate angles will be reported on in a forthcoming paper [14].

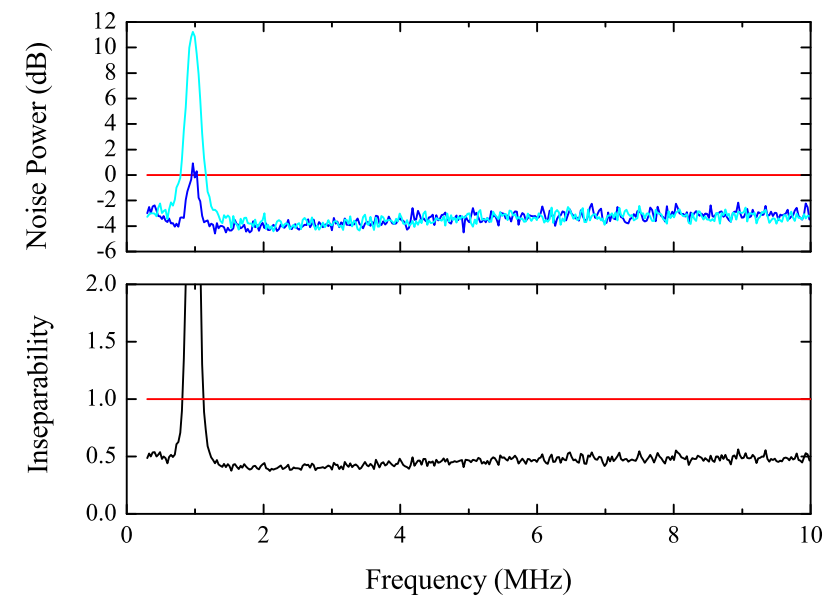

FIG. 2: Normalized noise variances from $300 \mathrm{kHz}$ to $10 \mathrm{MHz}$ of the $\pm 45^{\circ}$ vacuum modes and inseparability criterion for signal and idler modes defined as the half-sum of the previous squeezed variances. The resolution bandwidth is set to 100 $\mathrm{kHz}$ and the video bandwidth to $300 \mathrm{~Hz}$.

The experimental setup is shown in Figure 11 A continuous-wave frequency-doubled Nd:YAG laser ("Diabolo" without option "noise eater", Innolight $\mathrm{GmbH}$ ) pumps a triply resonant type-II phase-matched OPO, made of a semi-monolithic linear cavity : in order to increase the mechanical stability and reduce the reflection losses, the input flat mirror is directly coated on one face of the $10 \mathrm{~mm}$-long KTP crystal $\left(\theta=90^{\circ}, \varphi=23.5^{\circ}\right.$, Raicol Crystals Ltd.). The reflectivities for the input coupler are $95.5 \%$ for the pump at $532 \mathrm{~nm}$ and close to $100 \%$ for the signal and idler beams at $1064 \mathrm{~nm}$. The output coupler with a radius of curvature of $38 \mathrm{~mm}$ is highly reflecting for the pump and its transmission is $5 \%$ for the infrared. A birefringent plate $-\lambda / 4$ for the infrared and almost $\lambda$ for the pump - is inserted inside the cavity. Rotation as small as $0.01^{\circ}$ can be obtained thanks to a rotating mount controlled by a piezo-electric actuator. At exact triple resonance and for a very small angle of the plate relative to the axes of the crystal, the oscillation threshold is less than $20 \mathrm{~mW}$, close to the threshold without the plate [15]. The OPO is actively locked on the pump resonance by the Pound-Drever-Hall technique: we detect by reflection a $12 \mathrm{MHz}$ modulation and the error signal is sent to a home-made proportional-integral controller. The OPO can operate stably during more than one hour without mode-hopping using a drastic control of the crystal temperature within the $\mathrm{mK}$ range and an optical table isolated from floor vibrations by pneumatic feet (Newport I-2000).

The $1064 \mathrm{~nm}$ laser output is used as a local oscillator for homodyne detection of the generated state. This beam is spatially filtered and intensity-noise cleaned by 
a triangular-ring $45 \mathrm{~cm}$-long cavity with a high finesse of 3000 , which is locked on the maximum of transmission by the tilt-locking technique [16]. The homodyne detection is based on a pair of balanced high quantum efficiency InGaAs photodiodes (Epitaxx ETX300, quantum efficiency: 95\%) and the fringe visibility reaches 0.97 . The shot noise level of all measurements is easily obtained by blocking the output of the OPO. A half-wave plate inserted at the output of the OPO, just before the first polarizing beam splitter of the homodyne detection, enables us to choose the field to characterize: signal or idler modes which are entangled, or the $\pm 45^{\circ}$ rotated modes which are squeezed. The homodyne detection can be locked on the squeezed quadrature during more than an hour using a standard dither and lock technique. The error signal is extracted from the demodulated noise at a given frequency after enveloppe detection.

Figure 2 shows the experimental broadband noise reduction observed in the $\pm 45^{\circ}$ vacuum modes for frequencies between $300 \mathrm{kHz}$ and $10 \mathrm{MHz}$. One observes that these two modes are squeezed well-below the standard quantum limit, except around $1 \mathrm{MHz}$ where the narrow peak of excess noise is due to the relaxation oscillation of the laser. One can note that this excess is less important on the mode $A_{+}$which is sensitive to phase noise of the laser. A noise eater implemented on the Nd:YAG laser should permit to largely reduce this classical excess noise. The degree of entanglement between signal and idler fields can be evaluated by the inseparability criterion developed by Duan et al. [17] and Simon [18]: a necessary condition for inseparability is that the halfsum of the previous squeezed variances falls below one. This criterion is well verified in the considered frequency band as one can observe in Figure 2 To the best of our knowledge, our setup generates the best EPR entangled beams ever produced in the continuous variable regime. We have measured at a given noise frequency of $3.5 \mathrm{MHz}$ (RBW set to $100 \mathrm{kHz}$ and VBW to $300 \mathrm{~Hz}$ ) a value of the inseparability criterion equal to $0.33 \pm 0.02$ [14].

Figure 3sums up the same experimental measurements as given by Figure 2 but now for low noise frequencies, between $40 \mathrm{kHz}$ and $150 \mathrm{kHz}$. Measurements are corrected from the electronic dark noise which is at least $4 \mathrm{~dB}$ below all traces. One can see that the $\pm 45^{\circ}$ vacuum modes are strongly squeezed: the noise variances are still reduced by $3 \mathrm{~dB}$ around $100 \mathrm{kHz}$ and reach the shot noise limit for frequencies below $50 \mathrm{kHz}$. No significant difference is observed between the two rotated modes. The low limit frequency is well below the ones previously reported. In contrast to [8] and [9], the low frequency squeezing is obtained with the same efficiency on both rotated modes, showing that the effect is not due to common mode rejection of excess noise but to intrinsic absence of low frequency noise in our setup.

We have reported in this letter the demonstration of a compact source of squeezing from $50 \mathrm{kHz}$ to $10 \mathrm{MHz}$

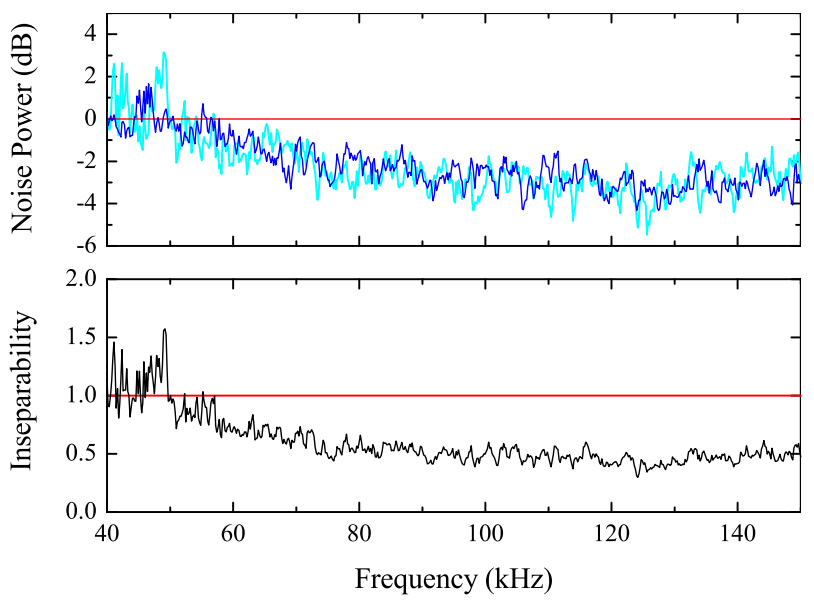

FIG. 3: Normalized noise variances from $40 \mathrm{kHz}$ to $150 \mathrm{kHz}$ of the $\pm 45^{\circ}$ vacuum modes after correction of the electronic noise and inseparability criterion for signal and idler modes. Squeezing and entanglement are observed down to $50 \mathrm{kHz}$. The resolution bandwidth is set to $3 \mathrm{kHz}$ and the video bandwidth to $10 \mathrm{~Hz}$.

(still present above, in the bandwidth of the cavity, but not measured) with a slight increase of noise in a frequency band of $100 \mathrm{kHz}$ around $1 \mathrm{MHz}$. This broadband noise reduction is likely to reduce noise in a pulsed measurement during a time window of duration T. The noise variance $\sigma^{2}$ of the measurement can be written

$$
\sigma^{2}=\int_{0}^{+\infty} S(\nu) T^{2} \operatorname{sinc}^{2}(\pi \nu T) d \nu
$$

where $S(\nu)$ is the spectral noise density of the light source. Let us evaluate the improvement obtained on a measurement during a window of duration $T=1 \mu \mathrm{s}$. We model our device by a shot-noise limited source below 50 $\mathrm{kHz}$ (the excess noise can be reduced to shot-noise by a feed-back loop) and squeezed by $3 \mathrm{~dB}$ above. In comparison with a shot-noise limited source at all frequencies, the noise variance is divided by a factor 1.7 . This very simple example shows the great interest of broadband and low-frequency squeezing to improve a large class of physical measurements.

In summary, we have demonstrated significant broadband vacuum EPR entanglement and squeezing down to $50 \mathrm{kHz}$ with a single OPO below threshold and without the need of optical noise cancellation technique. The degree of entanglement can be improved further by increasing the transmission escape efficiency of the OPO. Up to now, the attainable lowest frequency seems only limited by the technical noise of the laser and locking noise of the different cavities involved in our setup. The implementation of intensity noise eater on the Nd:YAG laser - as it is the case for gravitational wave detectors where lasers are expected to be shot noise limited around a few tens of $\mathrm{Hz}$ - should allow to reach even lower frequencies.

Laboratoire Kastler-Brossel, of the Ecole Normale 
Supérieure and the Université Pierre et Marie Curie, is associated with the Centre National de la Recherche Scientifique (UMR 8552). Laboratoire Matériaux et Phénomènes Quantiques is a Fédération de Recherche (CNRS FR 2437). This work has been supported by the European Commission project QUICOV (IST-199913071) and ACI Photonique (Ministère de la Recherche).

* Electronic address: coudreau@spectro.jussieu.fr

[1] C. M. Caves, Phys. Rev. D 23, 1693 (1981)

[2] P. Grangier, R. E. Slusher, B. Yurke, A. LaPorta, Phys. Rev. Lett. 59, 2153 (1987); P. Grangier, R. E. Slusher, B. Yurke, A. LaPorta, Proceedings NATO ASI Series B 190, 253 (1988); M. Xiao, L.A. Wu, H. J. Kimble, Phys. Rev. Lett. 59, 278 (1987)

[3] H.J. Kimble, Y. Levin, A. B. Matsko, K. S. Thorne, S. P. Vyatchanin, Phys. Rev. D 65, 022002 (2002); M. T. Jaekel, S. Reynaud, Europhys. Lett. 13, 301 (1990); W. G. Unruh, in Quantum Optics, Experimental Gravitation, and Measurement Theory, edited by P. Meystre and M. O. Scully (Plenum, New York, 1982), p. 647; A. F. Pace, M. J. Collett, D. F. Walls, Phys. Rev. A 47, 3173 (1993)

[4] K. McKenzie, D. A. Shaddock, D. E. McClelland, B. C. Buchler, P. K. Lam, Phys. Rev. Lett. 88, 231102 (2002)

[5] N. Treps, N. Grosse, W. P. Bowen, C. Fabre, H.-A Ba- chor, P. K. Lam, Science 301, 940 (2003)

[6] A. C. Boccara, D. Fournier, J. Badoz, Applied Phys. Lett. 36, 130 (1980)

[7] S. Feng, O. Pfister, J. Opt. B 5, 262 (2003).

[8] W. P. Bowen, R. Schnabel, N. Treps, H.-A Bachor, P. K. Lam, J. Opt. B 4, 421 (2002)

[9] R. Schnabel, H. Vahlbruch, A. Franzen, S. Chelkowski, N. Grosse, H.-A Bachor, W.P. Bowen, P. K. Lam, K. Danzmann, e-print quant-ph/0402064

[10] Quantum information with Continuous Variables, edited by S. L. Braunstein and A. K. Pati (Kluwer Academic Publishers, Dordrecht, 2003)

[11] E. J. Mason, N. C. Wong, Opt. Lett. 23, 1733 (1998)

[12] Z. Y. Ou, S. F. Pereira, H. J. Kimble, K. C. Peng, Phys. Rev. Lett. 68, 3663 (1992); Y. Zhang, H. Wang, X. Li, J. Jing, C. Xie, K. Peng, Phys. Rev. A, 62, 023813 (2000)

[13] L. Longchambon, J. Laurat, T. Coudreau, C. Fabre, submitted for publication in Eur. Phys. Journ. D, e-print quant-ph/0310036 and e-print quant-ph/0311123

[14] J. Laurat, L. Longchambon, G. Keller, T. Coudreau, N. Treps, C. Fabre, in preparation

[15] J. Laurat, T. Coudreau, N. Treps, A. Maître, C. Fabre, Phys. Rev. Lett. 91, 213601 (2003)

[16] D. A. Shaddock, M. B. Gray, D. E. McClelland, Opt. Lett. 24, 1499 (1999)

[17] L.-M. Duan, G. Giedke, J. I. Cirac, P. Zoller, Phys. Rev. Lett 84, 2722 (2000)

[18] R. Simon, Phys. Rev. Lett 84, 2726 (2000). 